\title{
Telemedicine and the risk of overshadowing children with Kawasaki disease in a worldwide coronavirus pandemic climate
}

\author{
Donato Rigante ${ }^{1,2}$ (1) \\ Received: 4 January 2021 / Accepted: 2 February 2021 / Published online: 13 February 2021 \\ c) Società Italiana di Medicina Interna (SIMI) 2021
}

\section{Dear Editor,}

I read with interest the paper by Molinari et al. [1], which appeared in Internal and Emergency Medicine 2020, dealing with the impact of severe acute respiratory syndrome coronavirus 2 (SARS-CoV-2) outbreak on the specific telemedicine management of cardiovascular diseases in Italy. The coronavirus disease 2019 (COVID-19) Public Health Emergency has caused an escalation of telemedicine services accompanied by governmental regulatory restrictions, and concurrently emergency department visits as well as hospital admissions were reduced at least for pediatric patients, due to the fear of contagions which discouraged many parents from seeking medical advice. Many weekly outpatients' evaluations were also discontinued and telephone consultations set up to assess children presenting fever during the past 2020 lockdown. Emergency Departments have been reorganized for implementing the efficiency of all diagnostic procedures required for COVID-19, but the risk of overshadowing severe non-COVID patients who might seek medical attention still persists.

Indeed, during the past year, a minority of persistentlyfebrile children have been shown to display features of multisystem inflammatory syndrome related to COVID-19, characterized by proinflammatory cytokine overproduction and features similar to Kawasaki disease (KD), one of the most relevant vasculitides of childhood and - of coursethe main cause of acquired heart disease in children living within the developed world [2]. A larger utilization of telemedicine should be warranted in a pandemic climate, but

Donato Rigante

donato.rigante@unicatt.it

1 Department of Life Sciences and Public Health, Fondazione Policlinico Universitario A. Gemelli IRCCS, Università Cattolica Sacro Cuore, Largo A. Gemelli 8, 00168 Rome, Italy

2 Università Cattolica del Sacro Cuore, Rome, Italy a far-flung distance cannot establish the right care relationship for children with KD, who are at risk of developing a future cardiovascular disease characterized by coronary artery aneurysms.

Unfortunately, KD diagnosis relies on the merely clinical recognition of few nonspecific clinical signs combined with undaunted persistent fever and is unlikely to verify via telemedicine services. The severity of KD and the risk of cardiovascular complications are proportional to the "extent" of systemic inflammation, requiring an urgent intervention with intravenous immunoglobulin (IVIG) aimed at reducing the inflammation in the coronary artery walls and preventing coronary artery thrombosis $[3,4]$. The potential role of viruses (like the SARS-CoV-2) entering through the upper respiratory tract as a trigger of systemic inflammation, which becomes "KD" in genetically-predisposed children, has been hypothesized since the first description of the disease, though a "second-hit" virus-mediated pathogenesis has been claimed by different authors [5].

The risk of missing KD diagnosis and overlooking treatment of KD for first-line providers who assess febrile children via telemedical devices might be relevant. The suspicion for KD should remain high in all children with prolonged fever, especially those younger than 1 year, and IVIG treatment should be given within 10 days from onset of fever to avoid a future surge of cardiovascular abnormalities in such patients. Early diagnosis of cardiovascular disease and cardiovascular rehabilitation are included in the goals of telemedicine, and remote telephone triaging might screen pediatric patients for the nature and urgency of the health care needs. Nevertheless, only physical examination can allow avoiding to eclipse children with KD. Telemedicine advocacy is a crucial resource during the COVID-19 pandemic, but a direct clinical assessment of children remains necessary in the acute care setting of KD, with opportunity to assess echocardiograms and ensure any involvement of the coronary arteries. 
Funding None.

\section{Compliance with ethical standard}

Conflict of interest The author declares that he has no conflict of interest.

Human/Animal rights The letter was prepared following the Declaration of Helsinki Ethical Principles for Medical Research Involving Human Subjects, adopted by the 18th World Medical Assembly, Helsinki, Finland, June 1964, and as amended most recently by the 64 th World Medical Assembly, Fontaleza, Brazil, October 2013.

Informed consent Written informed consent was not required, since the paper has no data obtained from patients.

\section{References}

1. Molinari G, Brunetti ND, Nodari S, Molinari M, Spagna G, Ioakim M, Migliore G, Dattoli V, Di Cillo O (2020) Impact of
2020 SARS-CoV-2 outbreak on telemedicine management of cardiovascular disease in Italy. Intern Emerg Med. https://doi. org/10.1007/s11739-020-02564-0

2. Bhat CS, Gupta L, Balasubramanian S, Singh S, Ramanan AV (2020) Hyperinflammatory syndrome in children associated with COVID-19: need for awareness. Indian Pediatr 57:929-935

3. Rigante D, Valentini P, Rizzo D, Leo A, De Rosa G, Onesimo R, De Nisco A, Angelone DF, Compagnone A, Delogu AB (2010) Responsiveness to intravenous immunoglobulins and occurrence of coronary artery abnormalities in a single-center cohort of Italian patients with Kawasaki syndrome. Rheumatol Int 30:841-846

4. Rigante D, Andreozzi L, Fastiggi M, Bracci B, Natale MF, Esposito S (2016) Critical overview of the risk scoring systems to predict non-responsiveness to intravenous immunoglobulin in Kawasaki syndrome. Int J Mol Sci 17:278

5. Rigante D (2020) Kawasaki disease as the immune-mediated echo of a viral infection. Mediterr J Hematol Infect Dis 12:e2020039

Publisher's Note Springer Nature remains neutral with regard to jurisdictional claims in published maps and institutional affiliations. 